\title{
Magnetic Refrigeration: emerging technology for sustainable refrigeration
}

\author{
Dorin Botoc ${ }^{1,2 *}$, Monica Siroux ${ }^{2}$, and Alexandru Salceanu ${ }^{3}$ \\ ${ }^{1}$ Gheorghe Asachi Technical University of Iasi, Romania \\ 2 INSA Strasbourg ICUBE, University of Strasbourg, 67000 Strasbourg, France \\ ${ }^{3}$ Department of Electrical Measurements and Electrotechnical Materials, Gheorghe Asachi Technical University of Iasi, Romania
}

\begin{abstract}
This article presents the magnetic refrigeration technology that is based on the magnetocaloric effect, heating or cooling of a magnetic material when the applied magnetic field changes. The first part of the paper shows an introductory study of the evolution of this technology over the years, continuing with the presentation of the magnetic regenerator model, which is composed of two heat exchangers with parallel plates of magnetocaloric material, gadolinium and are separated by heat transfer agent flow channels. The benefits and advantages of this technology are described, in the context of the current stage of development.
\end{abstract}

\section{Background and Introduction}

The technology to produce artificial cold based on vapor compression was introduced on the market, on an industrial scale, more than 120 years ago, with small improvements along the way. However, it has reached a technological level where improvements and performance increases of these types of installations are unlikely. Satisfying the growing demands of refrigeration and air conditioning at the same time, the reduction of greenhouse gases has led to the advancement of research and evolution of magnetic refrigeration systems to produce artificial cold. Magnetic refrigeration is based on the magnetocaloric effect of magnetocaloric materials that have this property, which manifests itself as a change in temperature when this material is subjected to a controlled magnetic field. Magnetic properties of magnetic materials have been known since antiquity and are used in various ways to obtain benefits from magnetic fields. The density of the magnetic flux initiates the process of electromagnetic induction in the case of receiver and transmitter coils, phenomena that are widely applied. Normally, the magnetocaloric effect is the property of magnetic materials where the process of temperature change, heating/cooling of the magnetic material takes place. The effect depends on the magnetic variation when the magnetic field is applied to the magnetocaloric material. Theoretically this explanation is based on magnetic property, magnetic entropy, and temperature. The potential for temperature change of magnetic materials that have magnetocaloric effect is huge, obviously the variation of temperature can be obtained based on the properties of magnetic materials. This effect is practically reversible, and when the material is no longer under the action of the magnetic field, it returns to its original state. The magnitude of the temperature change depends in most cases on the strength of the magnetic field and to obtain maximum energy efficiency and minimize the operating costs of this installation, it is recommended that the source of the magnetic field use permanent magnets. Magnetic refrigeration uses as a working agent, solid magnetocaloric materials, namely: silicon, gadolinium, prandliu etc. These materials illustrate the unique and most strongly highlighted property, which is called the magnetocaloric effect, and is manifested by increasing and decreasing the temperature of that material when it is magnetized or demagnetized, respectively.

This process of obtaining low temperatures, lower than those of the environment has as main purpose, thermal conditioning, industrial refrigeration, obtaining low temperatures, lower than those of the environment.

The comprehensive analysis of the literature was performed in this study to understand the main developments in this field. Moreover, the phenomena were also explained in detail to develop a much clearer and a clearer idea. All research has led to the development and real understanding of magnetism, temperature, and the properties of magnetocaloric materials. In [1] it explained the magnetic effect of Gd at 294K, and another researcher experimentally demonstrated low-temperature magnetic refrigeration facilities, explained the basic mechanism of the magnetic cooling cycle, and his fellow researchers argued and explained the need to develop new thermodynamic cycles to explain with certainty the phenomena that take place in magnetic refrigeration systems. Several authors have reported in many publications on the association of the Bryton and Ericsson cycle with magnetic refrigeration, [2] AMR (Active magnetic regenerator) operating on the Brayton cycle resulted in the production of the highest cooling capacity, while regenerator operating on the Ericsson cycle is the

* Corresponding author: dorinbotoc@yahoo.com

(c) The Authors, published by EDP Sciences. This is an open access article distributed under the terms of the Creative Commons Attribution License 4.0 
most efficient, [2] they also mathematically explained the numerical aspects of simulation.In [3] provided explanations based on the quantum of the magnetocaloric effect and proposed to increase the entropy in terms of the change in the magnetic field close to the quantum-critical points made magnetically by accurate and precise measurement of the calorimetric effect. The trend of research and publication in the field of magnetocaloric effect increased significantly until the end of the twentieth century due to the need for an energy efficient refrigeration and air conditioning system. The available scientific literature on the magnetocaloric effect can be divided into several types for specification, efficiency and relevance. These categories are based on materials, based on mathematics, experiment, numerical and thermodynamic.

The magnetocaloric effect used in magnetic refrigeration installations, has a wide use in this century of technology, namely, refrigeration systems, air conditioning, heat pumps, [4] aeronautical installations with direct application in the field of medicine, automotive and installations magnetic refrigeration appliances, household, etc., [5]. Fig. 1 shows the magnetization and demagnetization of the magnetocaloric material in isothermal and adiabatic processes.

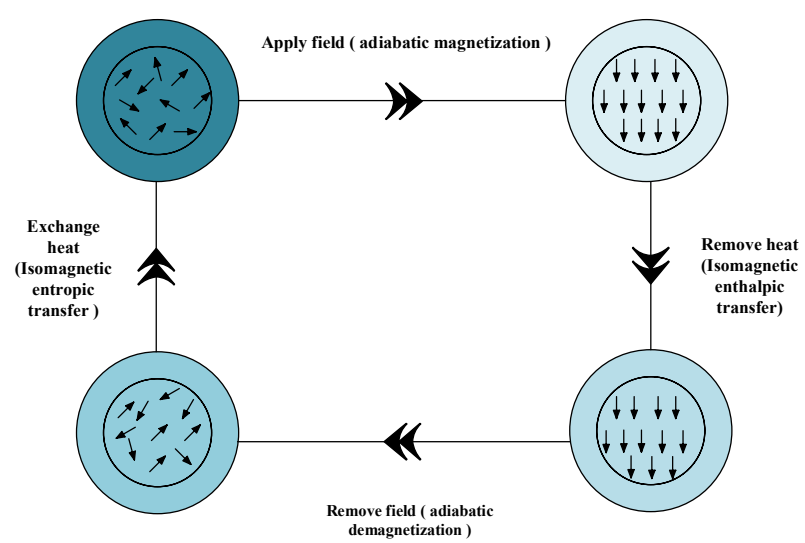

Fig. 1. The processes of magnetization and demagnetization of the magnetocaloric material, [8]

In [6], explained the magnetocaloric effect that is established in thermodynamics; and the variation of the primary state is the magnetic moment which is implicitly related to the magnetocaloric effect and the variables as internal energy representing the increase of temperature. The entropy of the system is in fact the magnetic entropy of the magnetocaloric effect. The magnetic field $\mathrm{H}$, applied from the outside leads to the adiabatic change of the temperature $\Delta \mathrm{Tad}$, being applied to a constant pressure of the heat transfer agent, [6]. The thermodynamic demonstration of the magnetocaloric effect was made by Pecharsky, and the compression of the working agent being isothermal and the decrease of the system entropy takes place when applying the pressure and being equivalent to the magnetization of the material and the magnetic part. The working agent distends adiabatically and when the pressure decreases, the temperature decreases, and this step is equivalent to the demagnetization of the magnetocaloric material which is removed from the magnetic field $\mathrm{H}$, [7]. The process is based on isothermal and adiabatic separation and the magnetic order can be seen in the figure above. With the adiabatic application of the magnetic field, there is a decrease in magnetic entropy. However, the total entropy of the entire system remains constant and as a result of this difference there is adiabatic growth, which can be explained as:

$$
\Delta T_{a d}=T_{1}-T_{0}
$$

Similarly, after the isothermal application of the magnetic field, the temperature must remain constant, but nevertheless we have a decrease in the entropy of the whole system due to the magnetization effect. The difference in entropy is represented by the change in the magnetic entropy of the whole system. $\Delta S_{m}$.

\section{Boundary condition, modelling and simulation}

Magnetocaloric refrigerators consist of a magnet, a regenerator made of a magnetocaloric material, a system that pumps a heat transfer fluid and two heat exchangers (cold (CHEX) and hot (HHEX) exchangers), [8-10]. The magnetic regenerator is the heart of the magnetic refrigeration system. We present here a magnetic regenerator 2D model. Fig. 2 shows the 2D model of the magnetic regenerator, which has a fluid channel length of $16,057 \mathrm{~cm}$ with a height of $3.1 \mathrm{~cm}$. The geometry of the regenerator is as follows: the width and height are equal to $5.2 \mathrm{~cm}$ and $1.7 \mathrm{~cm}$, respectively.

On either side of the regenerator are the cold (CHEX) and hot (HHEX) exchangers. The heat exchangers, CHEX and HHEX contain magnetocaloric material in the form of plates, and water was used as a heat transfer agent. The width and height of both heat exchangers are $1.8 \mathrm{~cm}$ and $2.5 \mathrm{~cm}$, respectively, [11-13].

The limit condition of the magnetic field used in this simulation is Ampere's law, while the condition of magnetization and demagnetization has been defined. The material used is solid in the $\mathrm{x}$ and $\mathrm{y}$ directions and the values of electrical conductivity and relative permeability were derived from the material parameters.

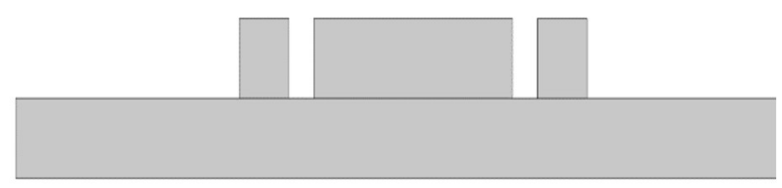

Fig. 2. 2D model of the magnetic regenerator, used in COMSOL Multiphysics software modelling

The heat transfer between the solid material and the heat transfer agent takes place in conditions of identification of the fluid and the solid domain, and the temperature has been defined, at the initial value of $293.15 \mathrm{~K}$. The normal inlet speed is $5 \mathrm{~m} / \mathrm{s}$ and was 
applied at the input, and at the output the zero-speed limitation condition was defined, Fig.3.

The proposed option offered by the solver is electromagnetic heating and isothermal conditions. The walls were modelled without limitation conditions.

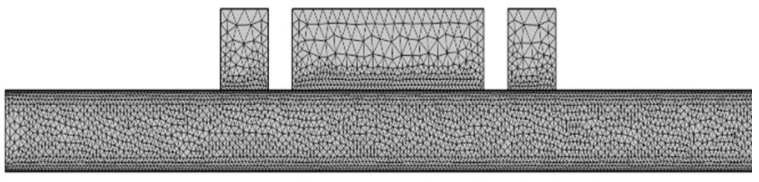

Fig. 3. The mesh model of the magnetic regenerator used in the COMSOL Multiphysics software simulation.

Maxwell equations can be used to represent the relationship between the magnetic field $H$, the temperature of the magnetocaloric material and its entropy, Ec.2.

$$
\left(\frac{\partial S(T, H)}{\partial H}\right)_{T}=\left(\frac{\partial M(T, H)}{\partial T}\right)_{H}
$$

For isothermal conditions, Ec. 2 can be integrated and obtained in the form of the equation below:

$$
\Delta S_{m}(T, \Delta H)=\int_{H_{1}}^{H_{2}}\left(\frac{\partial M(T, H)}{\partial T}\right) d H_{H}
$$

Table 1. Properties of the material used in the simulation of COMSOL Multiphysics software.

\begin{tabular}{|l|c|c|}
\hline Designation & Value & Unit \\
\hline Debye Temperature & 184 & $\mathrm{~K}$ \\
\hline Curie Temperature & 293 & $\mathrm{~K}$ \\
\hline Angular momentum & 3,5 & {$\left[\mathrm{~N}^{*} \mathrm{~m}^{*} \mathrm{~s}\right]$} \\
\hline Density & 7900 & {$\left[\mathrm{Kg} / \mathrm{m}^{\wedge} 3\right]$} \\
\hline Thermal coefficient & 0.0108784 & {$\left[\mathrm{~J} / \mathrm{mol}^{*} \mathrm{~K}^{\wedge} 2\right]$} \\
\hline
\end{tabular}

\section{Results and discussion}

The five basic components of the magnetic regenerator system are modelled explicitly and implicitly, [14]. The component that includes the magnetocaloric material is the only one explicitly modelled, while the magnetization system, the heat exchangers, and the pumping system of the working agent are modelled by default, Fig. 2 .

The temperature range of the magnetic regenerator is defined as the average temperature difference of the heat transfer fluid, the outlet between the "hot" and "cold" areas and the cooling and heating powers generated are defined using the following equations:

$$
\begin{aligned}
Q_{R}=f \int_{0}^{\tau_{f}} m_{f} c_{p, f} & \left(T_{f, c, \text { in }}(t)\right. \\
& \left.-T_{f, \text { c,out }}(t)\right) d t
\end{aligned}
$$

$$
\begin{aligned}
Q_{H}=f \int_{0}^{\tau_{f}} m_{f} c_{p, f} & \left(T_{f, h, \text { out }}(t)\right. \\
& \left.-T_{f, h, \text { in }}(t)\right) d t
\end{aligned}
$$

In Fig. 4. the results obtained in the MatLab environment are presented. The first figure represents the adiabatic temperature change of the gadolinium magnetocaloric material, used in the magnetic regenerator. The second figure shows specific magnetic entropy change, the third figure describes specific heat capacity while the last figure in this simulation shows the magnetization of gadolinium magnetocaloric material. It should be noted that this study was conducted to simulate the thermal processes in the magnetic regenerator, using the boundary condition of Ampere's law which was governed by the magnetic field module based on heat transfer due to the magnetocaloric effect and as a resultincrease temperature of the magnetocaloric material, gadolinium.

As can be seen in the Fig.4, there is magnetization nearby $T_{c}$ (temperature Curie) of the magnetocaloric material, gadolinium in the absence of the applied external magnetic field.
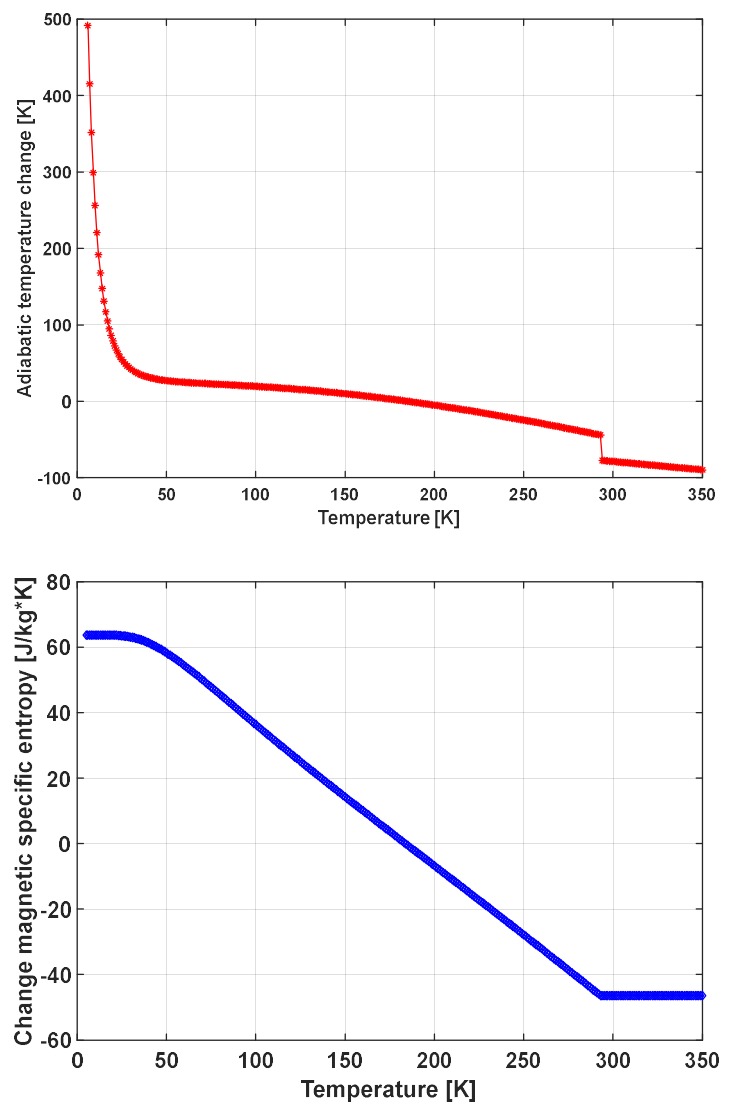

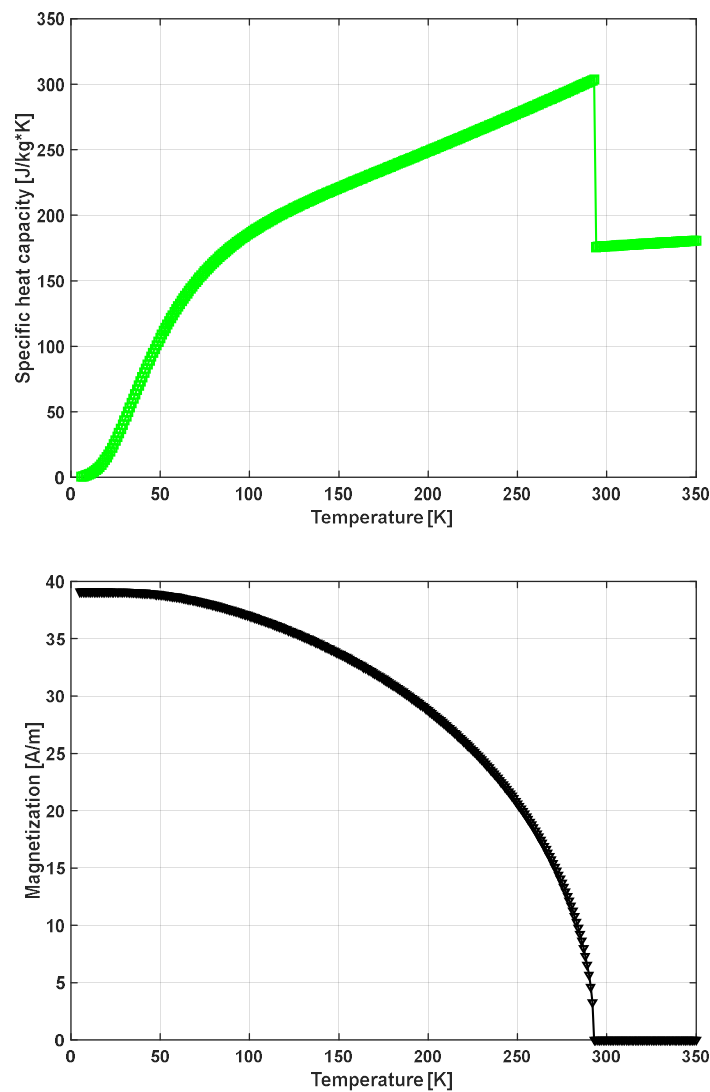

Fig. 4. The results obtained in the MatLab environment for adiabatic temperature change, change magnetic specific entropy, specific heat capacity and magnetization, [15].

Fig. 5 Shows the evolution of the regenerator temperature, [15-17]. The temperature evolution is similar in size to that reported in the literature. The maximum temperature range reached by the model is shown and presented the temperature difference between CHEX and HHEX over a period of 220 second.
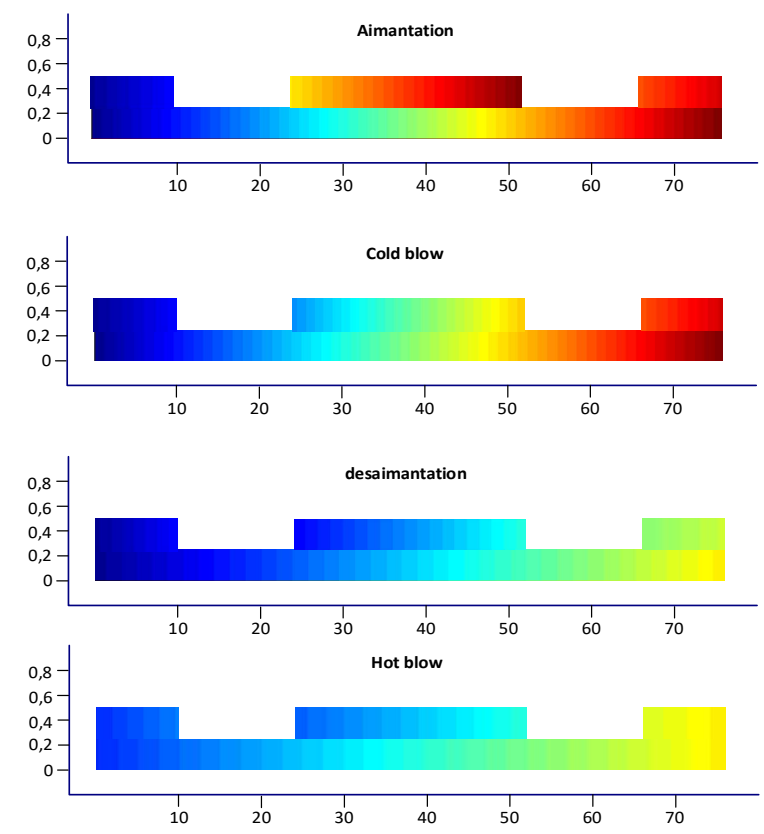

Fig. 5. 2D temperature evolution during a cycle
The refrigeration power is calculated according to the following relation:

$$
\dot{Q}_{\text {cold }}=\frac{1}{p} \int_{0}^{t_{\text {blow }}} \dot{M} \cdot c_{p, f}\left(T_{\text {cold }}-T_{f}(t)\right) d t
$$

The best performances are obtained at a frequency of $0.5 \mathrm{~Hz}$ with a fluid speed of 0.60 . The COP of the active magnetic regenerator system is calculated with the formula below:

$$
C O P=\frac{Q_{\text {cold }}}{W_{\text {mag }}+W_{\text {pump }}}
$$

The COP (coefficient of performance) decreases with the decrease of the volume of the heat transfer agent. The simulation began with the magnetization of the magnetocaloric material, using the boundary condition of Ampere's law, which was governed by the magnetic field modulus and the next step was based on heat transfer due to the magnetocaloric effect, resulting in increased temperature of the magnetocaloric material, gadolinium, the heat is transferred to the heat transfer agent and the final stage results in the release of thermal energy, which is controlled by the laminar flow module.

\section{Conclusions and perspective}

This paper describes the benefits and advantages of magnetic refrigeration in the current context of the development stage. The second part presents a numerical model of the magnetic regenerator, which is the subject of this study and its operation is at ambient temperature. In the third part of the paper are successfully presented the results obtained, which validates the proposed model of the magnetic regenerator, which works based on the magnetocaloric effect.

\section{References}

1. V Pecharsky, K Gschneidner Jr, "Magnetocaloric effect and magnetic refrigeration," Journal of Magnetism and Magnetic Materials, vol. 200, no. 13, pp. 44-56, (1999)

2. A Kitanovski, U Plaznic, J Tusek, A Poredos, "New thermodinamic cycles for magnetic refrigeration," International Journal of Refrigeration, vol. 37, pp. 2835, (2014)

3. B Wolf et al, "Magnetocaloric effect and magnetic cooling near a field induced quantum critical point," National Academy Sciences, vol. 108, no. 17, pp. 6862-6866, (2011)

4. A Kitanovski, "Energy Applications of Magnetocaloric," Advanced Energy Materials, vol. 10 , no. 10, (2020)

5. P Shirron, "Applications of the magnetocaloric effect in single stage, multi stage and continuos adiabatic demagnetization refrigerators," Cryogenics, vol. 62, pp. 130-139, (2014) 
6. V Basso, "Basics of the magnetocaloric effect," (2013)

7. V Pecharsky, K Gschneidner Jr, "Giant Magnetocaloric Effect in Gd5(Si2Ge2)," Physical Reciew Letters, vol. 78, pp. 4494-4497, (1997)

8. Botoc Dorin, Jurij Avsec, Adrian Plesca, "The efficiency of magnetic refrigeration and a comparison with compressor refrigeration systems," Journal of Energy Technology, vol. 11, pp. 59-69, (2018)

9. A Tusek, A Kitanovski, S Zupan et al , „A comprehensive experimental analysis of gadolinium active magnetic regenerators," Appl Therm Eng, nr. 53:57-66, (2013)

10. B Dorin, J Avsec, A Plesca, "A regenerator used in the installation of magnetic refrigeration devices," Journal of Energy Technology, vol. 13, nr. 3, pp. 57$66,(2020)$

11. J Tusek, A Kitanovski, I Prebil et al, „Dynamic operation of an active magnetic regenerator (AMR): numerical optimization of a packed-bed AMR," Int J Refrig, nr. 34:1507-1517, (2011)
12. T D Alan Nakashima, F P Fortkamp, M Natalia et al, „A magnetic wine cooler prototyp," Int J Refrig, vol. 122, pp. 110-121, (2021)

13. A Tura, A Rowe, „Permanent magnet magnetic refrigerator design and experimental characterization," Int J Refrig, nr. 34(3):628-639, (2011)

14. DS Arnold, A Tura, A Ruebsaat-Trott et al, „Design improvements of a permanent magnet active magnetic refrigerator," Int J Refrig, nr. 37:99-105, (2014)

15. S Lionte, C Vasile, M Siroux, ,Numerical analysis of a reciprocating active magnetic regenerator," App Ther Engineering, pp. 1-9, 2014

16. V Basso, CP Sasso, G Bertotti et al, „Effect of material hysteresis in magnetic refrigeration cycle," Int J Refrig, nr. 29:1358-1365, (2006)

17. A Kitanovski, U Plaznic, J Tusek et al, „New thermodinamic cycle for magnetic refrigeration," Int J Refrig, nr. 37:28-35, (2014) 